\title{
Regular homogeneously traceable nonhamiltonian
} graphs*

\author{
Yanan $\mathrm{Hu}$ and Xingzhi Zhan ${ }^{\dagger}$ \\ Department of Mathematics, East China Normal University, Shanghai 200241, China
}

\begin{abstract}
A graph is called homogeneously traceable if every vertex is an endpoint of a Hamilton path. In 1979 Chartrand, Gould and Kapoor proved that for every integer $n \geq 9$, there exists a homogeneously traceable nonhamiltonian graph of order $n$. The graphs they constructed are irregular. Thus it is natural to consider the existence problem of regular homogeneously traceable nonhamiltonian graphs. We prove two results: (1) For every even integer $n \geq 10$, there exists a cubic homogeneously traceable nonhamiltonian graph of order $n$; (2) for every integer $p \geq 18$, there exists a 4-regular homogeneously traceable graph of order $p$ and circumference $p-4$. Unsolved problems are posed.
\end{abstract}

Key words. Homogeneously traceable; regular graph; circumference Mathematics Subject Classification. 05C38, 05C45, 05C76

\section{Introduction}

We consider finite simple graphs. The order of a graph is its number of vertices, and the size is its number of edges. We denote by $V(G)$ the vertex set of a graph $G$. The following concept is introduced by Skupien in 1975 (see [3, p.185], and [4]). Note that the preprint of the 1984 paper [4] was cited by the 1979 paper [2].

${ }^{*}$ E-mail addresses: huyanan530@163.com(Y.Hu), zhan@math.ecnu.edu.cn(X.Zhan).

${ }^{\dagger}$ Corresponding author. 
Definition 1. A graph $G$ is said to be homogeneously traceable if every vertex of $G$ is an endpoint of a Hamilton path.

Obviously, hamiltonian graphs and hypohamiltonian graphs are homogeneously traceable. Chartrand, Gould and Kapoor [2] proved that for every integer $n$ with $3 \leq n \leq 8$, any homogeneously traceable graph of order $n$ is hamiltonian and that for $n \geq 9$, there exists a homogeneously traceable nonhamiltonian graph of order $n$. This result was rediscovered in [1] where the term "homogeneously traceable" was called "fully strung". The homogeneously traceable nonhamiltonian graphs constructed in [2] are irregular while the homogeneously traceable nonhamiltonian graphs constructed in [1] are also irregular except the Petersen graph of order 10 which is cubic (i.e., 3-regular). Thus it is natural to consider the existence problem of regular homogeneously traceable nonhamiltonian graphs.

In Section 2 we construct regular homogeneously traceable nonhamiltonian graphs, and in Section 3 we pose two unsolved problems.

\section{Regular homogeneously traceable nonhamiltonian graphs}

Given a vertex $v$ in a graph, a $v$-path is a path with $v$ as an endpoint. We use $K_{d}$ to denote the complete graph of order $d$, and use $N(v)$ to denote the neighborhood of a vertex $v$. The notation $\operatorname{circum}(G)$ means the circumference of a graph $G$.

Definition 2. Let $v$ be a vertex of degree $d$ in a graph. Blowing up $v$ into the complete graph $K_{d}$ is the operation of replacing $v$ by $K_{d}$ and adding $d$ edges joining the vertices of $K_{d}$ to the vertices in $N(v)$ such that the new edges form a matching.

The operation of blowing up a vertex of degree 4 into $K_{4}$ is depicted in Figure 1. 


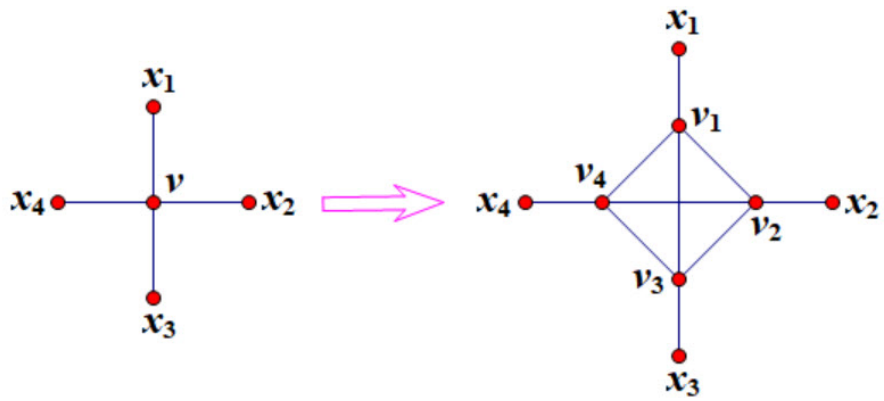

Fig. 1. Blowing up v into $\mathrm{K}_{4}$

Definition 3. A graph $G$ is called doubly homogeneously traceable if for any vertex $v$ of $G$, there are two Hamilton $v$-paths $P$ and $Q$ such that the two edges incident to $v$ on $P$ and $Q$ are distinct.

We will need the following two lemmas.

Lemma 1. Let $v$ be a vertex of degree 3 in a doubly homogeneously traceable graph $G$ of order $n$ and circumference $c$. Suppose $G^{\prime}$ is the graph obtained from $G$ by blowing up $v$ into $K_{3}$. Then $G^{\prime}$ is also doubly homogeneously traceable. If $v$ lies in a longest cycle of $G$, then $G^{\prime}$ has circumference $c+2$.

Proof. Let $N(v)=\left\{x_{1}, x_{2}, x_{3}\right\}$ and suppose $v$ is blown up into $K_{3}$ whose vertices are $v_{1}, v_{2}, v_{3}$ such that $v_{i}$ is adjacent to $x_{i}$ for $i=1,2,3$. Let $u \in V\left(G^{\prime}\right)$. If $u \notin\left\{v_{1}, v_{2}, v_{3}\right\}$, there exist two Hamilton $u$-paths $P: u, \ldots, x_{i}, v, x_{j}, \ldots$ and $Q: u, \ldots, x_{s}, v, x_{t}, \ldots$ of $G$ where the two edges incident to $u$ on $P$ and $Q$ are distinct. Then $G^{\prime}$ has two Hamilton $u$ paths $u, \ldots, x_{i}, v_{i}, v_{f}, v_{j}, x_{j}, \ldots$ and $u, \ldots, x_{s}, v_{s}, v_{g}, v_{t}, x_{t}, \ldots$ where the two edges incident to $u$ are distinct.

Next suppose $u \in\left\{v_{1}, v_{2}, v_{3}\right\}$. Without loss of generality suppose $G$ has two Hamilton $v$-paths $v, x_{1}, \ldots$ and $v, x_{2}, \ldots$ Then $G^{\prime}$ has two Hamilton $v_{3}$-paths: $v_{3}, v_{2}, v_{1}, x_{1}, \ldots$ and $v_{3}, v_{1}, v_{2}, x_{2}, \ldots$ Since $G$ is doubly homogeneously traceable, $G$ has a Hamilton $x_{1^{-}}$ path $x_{1}, y, \ldots, x_{i}, v, x_{j}, \ldots$ with $y \neq v$. It follows that $G^{\prime}$ has two Hamilton $v_{1}$-paths: $v_{1}, x_{1}, y, \ldots, x_{i}, v_{i}, v_{j}, x_{j}, \ldots$ and $v_{1}, v_{3}, v_{2}, x_{2}, \ldots$, where the two edges $v_{1} x_{1}$ and $v_{1} v_{3}$ are distinct. Similarly we can show that $G^{\prime}$ has two Hamilton $v_{2}$-paths where the two edges incident to $v_{2}$ are distinct. This completes the proof that $G^{\prime}$ is doubly homogeneously traceable.

Now suppose $v$ lies in a longest cycle of $G$. Let $\ldots, x_{i}, v, x_{j}, \ldots$ be a cycle of $G$ with length $c$. Then $G^{\prime}$ contains the cycle $\ldots, x_{i}, v_{i}, v_{f}, v_{j}, x_{j}, \ldots$ which has length $c+2$. Thus $\operatorname{circum}\left(G^{\prime}\right) \geq c+2$. On the other hand, let $C$ be a longest cycle of $G^{\prime}$, which has length at 
least $c+2$. If $C$ contains no vertex from the set $S=\left\{v_{1}, v_{2}, v_{3}\right\}$, it is also a cycle in $G$ and hence has length at most $c$, a contradiction. Observe that every vertex in $S$ has exactly one neighbor outside $S$. If $C$ contains a vertex in $S$, then $C$ contains at least two vertices in $S$. Note that the vertices in $V(C) \cap S$ appear consecutively on $C$. Since $S$ is a clique and $C$ is a longest cycle, we deduce that $|V(C) \cap S|=3$. Thus $v_{r} v_{s} v_{t}$ is a path on $C$ with $\{r, s, t\}=\{1,2,3\}$. Replacing this path by the vertex $v$ we obtain a cycle in $G$ which has length at most $c$. Hence $C$ has length at most $c+2$, implying that $\operatorname{circum}\left(G^{\prime}\right) \leq c+2$. Combining this inequality with $\operatorname{circum}\left(G^{\prime}\right) \geq c+2$ we obtain $\operatorname{circum}\left(G^{\prime}\right)=c+2$.

Lemma 2. Let $v$ be a vertex of degree 4 in a doubly homogeneously traceable graph $G$ of order $n$ and circumference $c$. Suppose $G^{\prime}$ is the graph obtained from $G$ by blowing up $v$ into $K_{4}$. Then $G^{\prime}$ is also doubly homogeneously traceable. If $v$ lies in a longest cycle of $G$ and $v$ lies in a clique of cardinality 4 , then $G^{\prime}$ has circumference $c+3$ and $G^{\prime}$ contains a vertex $v^{\prime}$ that lies in a longest cycle of $G^{\prime}$ and also lies in a clique of cardinality 4.

Proof. The proof that $G^{\prime}$ is doubly homogeneously traceable is similar to that in the above proof of Lemma 1 (but easier).

Next suppose $v$ lies in a longest cycle of $G$ and $v$ lies in a clique of cardinality 4 . Let $N(v)=\left\{x_{1}, x_{2}, x_{3}, x_{4}\right\}$ where $v, x_{1}, x_{2}, x_{3}$ form a clique and suppose $v$ is blown up into $K_{4}$ whose vertices are $v_{1}, v_{2}, v_{3}, v_{4}$ such that $v_{i}$ is adjacent to $x_{i}$ for $i=1,2,3,4$. See Figure 2 for the change of local structures.

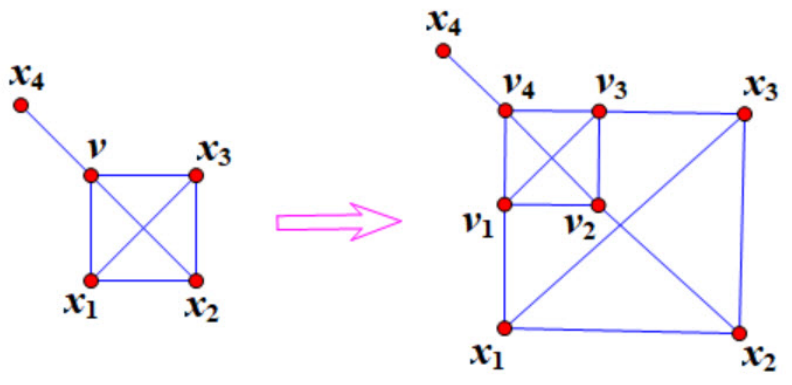

Fig. 2. Local changes

Let $\ldots, x_{i}, v, x_{j}, \ldots$ be a longest cycle of $G$ with length $c$. Then $G^{\prime}$ contains the cycle $\ldots, x_{i}, v_{i}, v_{s}, v_{t}, v_{j}, x_{j}, \ldots$ which has length $c+3$. Thus $\operatorname{circum}\left(G^{\prime}\right) \geq c+3$. We then prove the reverse inequality. Let $C$ be a longest cycle of $G^{\prime}$, which has length at least $c+3$. Denote $S=\left\{v_{1}, v_{2}, v_{3}, v_{4}\right\}$. If $C$ contains no vertex from the set $S$, it is also a cycle in $G$ and hence has length at most $c$, a contradiction. Note that every vertex in $S$ has exactly one neighbor outside $S$. Thus, if a cycle contains a vertex in $S$, it contains at least two. We have $|V(C) \cap S| \geq 2$. If $w \in V(C) \cap S$, then at least one neighbor of $w$ on $C$ belongs to $S$. 
Since $S$ is a clique and $C$ is a longest cycle, we deduce that $|V(C) \cap S|=4$. On the cycle $C$, using the vertex $v$ instead of $v_{4}$ and replacing a path of length 5 by a path of length 2 , or replacing two paths of length 3 and 2 respectively by two edges we obtain a cycle of $G$, where we have used the fact that $v, x_{1}, x_{2}, x_{3}$ form a clique in $G$. Since $\operatorname{circum}(G)=c$, the cycle $C$ has length at most $c+3$. This proves $\operatorname{circum}\left(G^{\prime}\right)=c+3$.

Finally we may choose $v_{4}$ as the vertex $v^{\prime}$.

Now we are ready to state and prove the main results.

Theorem 3. For every even integer $n \geq 10$, there exists a cubic homogeneously traceable nonhamiltonian graph of order $n$; for every integer $p \geq 18$, there exists a 4 regular homogeneously traceable graph of order $p$ and circumference $p-4$.

Proof. The Petersen graph $P$ depicted in Figure 3 is a cubic doubly homogeneously traceable graph of order 10 and circumference 9 .

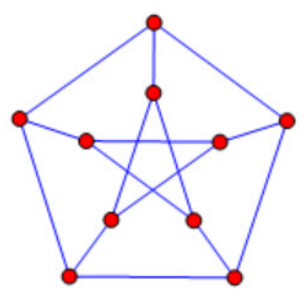

Fig. 3. The Petersen graph

Note that every vertex of $P$ lies in a longest cycle. Thus, choosing any vertex $v$ of $P$ and blowing up $v$ into $K_{3}$ we obtain a cubic graph $P_{12}$ of order 12 . By Lemma $1, P_{12}$ is doubly homogeneously traceable and has circumference 11 . Let $u$ be a vertex of $P_{12}$ that lies in a longest cycle. In $P_{12}$, blowing up $u$ into $K_{3}$ we obtain a cubic graph $P_{14}$ of order 14. By Lemma 1, $P_{14}$ is doubly homogeneously traceable and has circumference 13. Continuing this process we can construct a cubic homogeneously traceable graph of order $n$ and circumference $n-1$ for any even integer $n \geq 10$.

It is easy to verify that the three graphs in Figures 4, 5 and 6 are 4-regular doubly homogeneously traceable graphs of order $p$ and circumference $p-4$ for $p=18,19,20$ respectively, where the vertices $x, y, z$ lie in a longest cycle and in a clique of cardinality 4. 


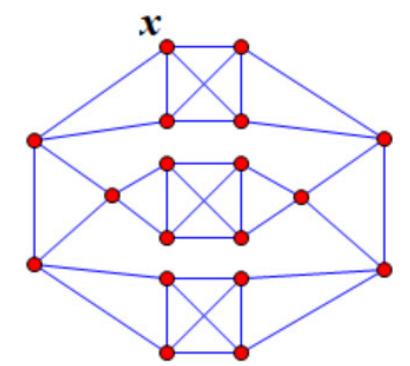

Fig. 4. The 4-regular base graph of order 18

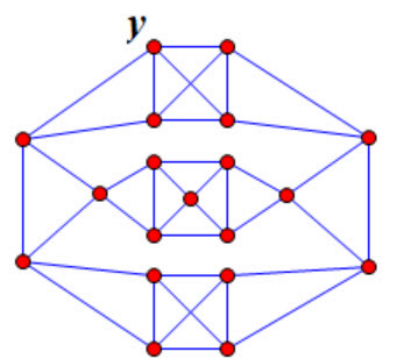

Fig. 5. The 4-regular base graph of order 19

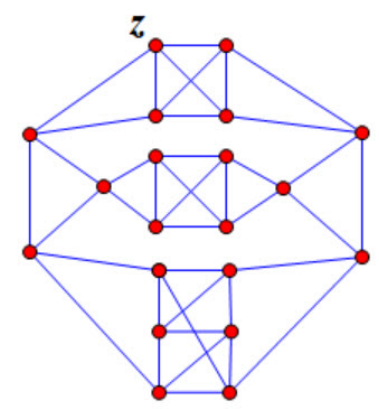

Fig. 6. The 4-regular base graph of order 20

Next we apply Lemma 2 repeatedly. Starting from the graph in Figure 4 and the vertex $x$, successively blowing up a vertex that lies in a longest cycle and in a clique of cardinality 4, we can construct a 4-regular homogeneously traceable graph of order $p$ and circumference $p-4$ for every integer $p \geq 18$ with $p \equiv 0 \bmod 3$. Starting from the graph in Figure 5 and the vertex $y$, successively blowing up a vertex that lies in a longest cycle and in a clique of cardinality 4, we can construct a 4-regular homogeneously traceable graph of order $p$ and circumference $p-4$ for every integer $p \geq 19$ with $p \equiv 1 \bmod 3$. Starting from the graph in Figure 6 and the vertex $z$, successively blowing up a vertex that lies in a longest cycle and in a clique of cardinality 4, we can construct a 4-regular homogeneously traceable graph of order $p$ and circumference $p-4$ for every integer $p \geq 20$ with $p \equiv 2 \bmod 3$. This completes the proof. 
Remark. The above proof of Theorem 3 shows that in the statement of Theorem 3 , we may replace "homogeneously traceable" by "doubly homogeneously traceable". But we prefer the current version, since the term "doubly homogeneously traceable" is technical in some sense.

\section{$3 \quad$ Unsolved problems}

It is known ([2, Theorem 4] and [4, pp. 9-11]) that the minimum size of a homogeneously traceable nonhamiltonian graph of order $n$ is $\lceil 5 n / 4\rceil$.

The extremal problem concerning the independence number is easy.

Theorem 4. The maximum independence number of a homogeneously traceable graph of order $n$ is $\lfloor n / 2\rfloor$.

Proof. Let $G$ be a homogeneously traceable graph of order $n$, and let $v_{1}, v_{2}, \ldots, v_{n}$ be a Hamilton path. Suppose $S$ is an independent set of $G$. If $n$ is even, $S$ contains at most one vertex in each of the edges $v_{1} v_{2}, v_{3} v_{4}, \ldots, v_{n-1} v_{n}$ and hence $|S| \leq n / 2$. Now suppose $n$ is odd. Similarly, we have $|S| \leq(n+1) / 2$. We will show that $|S|$ cannot equal $(n+1) / 2$. To the contrary, assume $|S|=(n+1) / 2$. Then $S=\left\{v_{1}, v_{3}, v_{5}, \ldots, v_{n}\right\}$. Since $G$ is homogeneously traceable, there is a Hamilton path $v_{2}, v_{i_{2}}, \ldots, v_{i_{n}}$. Since $n$ is odd, there exists an integer $k$ with $2 \leq k \leq n-1$ such that both $i_{k}$ and $i_{k+1}$ are odd. But $v_{i_{k}}$ and $v_{i_{k+1}}$ are adjacent and they both belong to $S$, contradicting the condition that $S$ is an independent set. It follows that $|S| \leq(n-1) / 2$. We have proved that $|S| \leq\lfloor n / 2\rfloor$.

This upper bound $\lfloor n / 2\rfloor$ can be attained by the cycle $C_{n}$ which is homogeneously traceable, and hence it is indeed the maximum value.

Finally we pose two unsolved problems.

Conjecture 1. The minimum circumference of a homogeneously traceable graph of order $n$ is $\lceil 2 n / 3\rceil+2$.

The circumference $\lceil 2 n / 3\rceil+2$ in Conjecture 1 is attained by the graph in Figure 7 where $p=\lfloor(n-6) / 3\rfloor$ and when $p \geq 2$ the vertices $u$ and $v$ are distinct, $x$ and $y$ are distinct and $w$ and $z$ are distinct. 


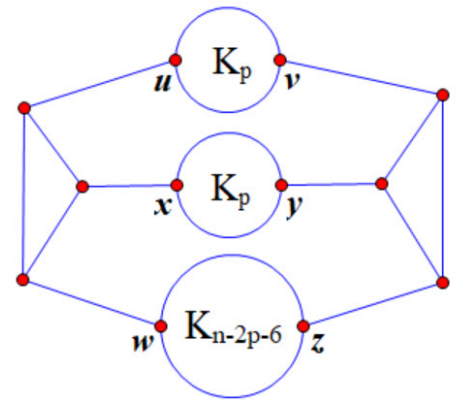

Fig. 7. A homogeneously traceable graph of a small circumference

Problem 2. Given an integer $k \geq 4$, determine the integers $n$ such that there exists a k-regular homogeneously traceable nonhamiltonian graph of order $n$.

Theorem 3 solves the case $k=3$ of Problem 2. A computer search shows that there exists no 4-regular homogeneously traceable nonhamiltonian graph of order $\leq 15$. Thus, according to Theorem 3, only the two orders 16 and 17 are uncertain for $k=4$.

Acknowledgement. This research was supported by the NSFC grants 11671148 and 11771148 and Science and Technology Commission of Shanghai Municipality (STCSM) grant 18dz2271000.

\section{References}

[1] E. Carlson, W. Fletcher and M. Montee, Hamiltonian paths in non-hamiltonian graphs, arXiv: 2106.13372v1, 25 June 2021.

[2] G. Chartrand, R.J. Gould and S.F. Kapoor, On homogeneously traceable nonhamiltonian graphs, 2nd International Conference on Combinatorial Mathematics, Ann. N.Y. Acad. Sci., 319(1979), 130-135.

[3] Z. Skupień, Degrees in homogeneously traceable graphs, Ann. Discrete Math., 8(1980), 185-188.

[4] Z. Skupień, Homogeneously traceable and hamiltonian-connected graphs, Demonstratio Math., 17(1984), no.4, 1051-1067. 